Rui Duan, Hui Tian, Yu Guojun and Qiong Wu*

\title{
Crystal structure of azido- $k^{1} N-\left\{6,6^{\prime}-\right.$ ((((methylazanediyl)bis(propane-3,1-diyl)) bis(azanylylidene))bis(methanylylidene))bis(2,4- dibromophenolato) $\left.k^{5} N, N^{\prime}, N^{\prime \prime}, 0,0^{\prime}\right\}$ cobalt(III)- methanol (1/1)), $\mathrm{C}_{21} \mathrm{H}_{23} \mathrm{Br}_{4} \mathrm{CoN}_{6} \mathrm{O}_{3}$
}

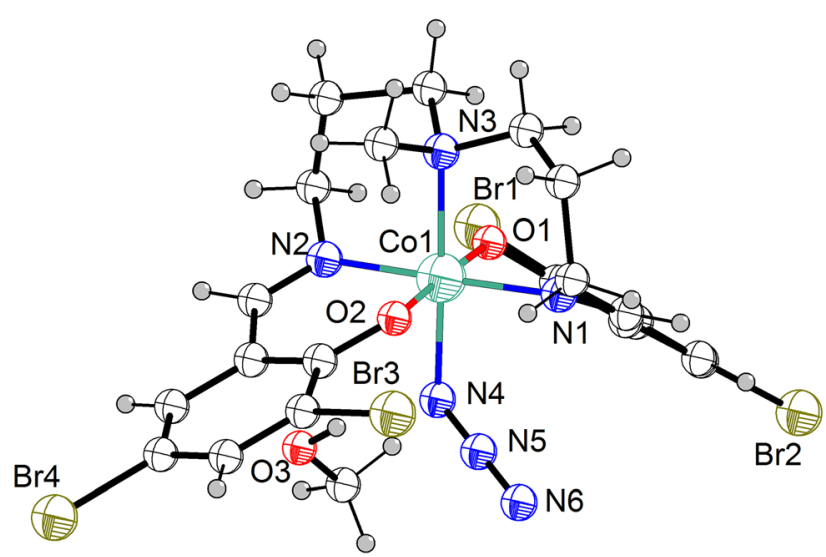

https://doi.org/10.1515/ncrs-2021-0375

Received September 29, 2021; accepted November 8, 2021; published online November 17, 2021

\begin{abstract}
$\mathrm{C}_{21} \mathrm{H}_{23} \mathrm{Br}_{4} \mathrm{CoN}_{6} \mathrm{O}_{3}$, monoclinic, $P 2_{1} / c$ (no. 14), $a=16.2300(4) \AA, b=20.9661(6) \AA, c=7.8269(2) \AA$, $\beta=97.2250(11)^{\circ}, V=2642.19(12) \AA^{3}, Z=4, R_{g t}(F)=0.0336$, $w R_{\text {ref }}\left(F^{2}\right)=0.0981, T=150.0 \mathrm{~K}$.
\end{abstract}

CCDC no.: 2112998

The molecular structure is shown in the figure. Table 1 contains crystallographic data and Table 2 contains the list of the atoms including atomic coordinates and displacement parameters.

\footnotetext{
*Corresponding author: Qiong Wu, Department of Chemical Science and Technology, Kunming University, Kunming, Yunnan 65200, P. R. China, E-mail: wuqiongkm@163.com. https://orcid.org/0000-00017931-6750

Rui Duan, Hui Tian and Yu Guojun, Department of Chemical Science and Technology, Kunming University, Kunming, Yunnan 65200, P. R. China
}

Table 1: Data collection and handling.

\begin{tabular}{ll}
\hline Crystal: & Black block \\
Size: & $0.22 \times 0.20 \times 0.18 \mathrm{~mm}$ \\
Wavelength: & Mo $K \alpha$ radiation $(0.71073 \AA)$ \\
$\mu:$ & $6.73 \mathrm{~mm}^{-1}$ \\
Diffractometer, scan mode: & Xcalibur Eos, $\varphi$ and $\omega$ \\
$\theta_{\text {max }}$, completeness: & $26.4^{\circ}, 99 \%$ \\
$N\left(h k l_{\text {measured }}, N(h k l)_{\text {unique }}, R_{\text {int }}:\right.$ & $37,075,5358,0.045$ \\
Criterion for $I_{\text {obs }}, N(h k l)_{\text {gt }}:$ & $I_{\text {obs }}>2 \sigma\left(I_{\text {obs }}\right), 4612$ \\
$N(\text { param })_{\text {refined: }}$ & 318 \\
Programs: & CrysAlis \\
& SHEL $[1]$, Olex2 [2], $]$ \\
\hline
\end{tabular}

\section{Source of material}

$N^{\prime}, N$-bis(3-aminopropyl) methylamine (0.81 $\left.\mathrm{mL}, 5 \mathrm{mmol}\right)$ was added into an anhydrous ethanol solution $(40 \mathrm{~mL})$ of 3,5-bromosalicylaldehyde (1.90 g, $10 \mathrm{mmol})$. The solution was refluxed at $60^{\circ} \mathrm{C}$ for ca. $3 \mathrm{~h}$, the yellow suspension was then stirred at $60{ }^{\circ} \mathrm{C}$ for $30 \mathrm{~min}$. Then, $\mathrm{Co}\left(\mathrm{NO}_{3}\right)_{2} \cdot 6 \mathrm{H}_{2} \mathrm{O}$ ( $0.145 \mathrm{~g}, 0.5 \mathrm{mmol}$ ) was added and the resulting brown mixture was further stirred for another $30 \mathrm{~min}$ and filtered. The black crystals of the title compound were obtained after one week by slow evaporation.

\section{Experimental details}

The crystal sample was selected by microscope and mounted on a glass fiber. Structure solution and refinements were carried out using the Olex2 software package [2]. All the hydrogen atoms were theoretically placed onto the ligand and refined using the riding model approximation. For the $\mathrm{CH}_{2}$-groups, $\mathrm{C}-\mathrm{H}$ bonds length were fixed at $0.97 \AA$ and for the aromatic $\mathrm{C}-\mathrm{H}$ distances $0.93 \AA$ was used. 
Table 2: Fractional atomic coordinates and isotropic or equivalent isotropic displacement parameters $\left(\AA^{2}\right)$.

\begin{tabular}{|c|c|c|c|c|}
\hline Atom & $x$ & $y$ & $z$ & $U_{\text {iso }} * / U_{\text {eq }}$ \\
\hline$r 1$ & $0.35748(3)$ & $0.70775(2)$ & $-0.06633(6)$ & $0.0417(12)$ \\
\hline 12 & $0.04508(3)$ & $0.71859(2)$ & $-0.47685(6)$ & 0.05029 (13) \\
\hline r3 & $0.15392(3)$ & $0.31527(2)$ & $0.34082(6)$ & 0.04569 (13) \\
\hline 14 & $0.44511(3)$ & $0.20401(3)$ & $0.15173(8)$ & $0.06241(16)$ \\
\hline $00 \mathrm{~A}$ & $0.25949(3)$ & $0.51921(2)$ & $0.19441(6)$ & $0.02955(12)$ \\
\hline 01 & $0.27174(16)$ & 0.60307 (13) & $0.1100(3)$ & $0.0352(6)$ \\
\hline 02 & $0.24622(16)$ & $0.43650(12)$ & $0.2831(3)$ & $0.0338(6)$ \\
\hline N1 & 0.14114 (19) & $0.53000(15)$ & $0.1745(4)$ & $0.0316(7)$ \\
\hline N2 & $0.2715(2)$ & $0.55001(17)$ & $0.4342(4)$ & $0.0385(7)$ \\
\hline $\mathrm{H} 2$ & 0.295521 & 0.512652 & .502650 & $0.046^{*}$ \\
\hline N3 & $0.37812(19)$ & $0.50775(17)$ & $0.2161(4)$ & $0.0348(7)$ \\
\hline N4 & 0.24707 (19) & $0.48515(16)$ & $-0.0407(4)$ & $0.0341(7)$ \\
\hline$\sqrt{5}$ & $0.3028(2)$ & $0.48924(19)$ & $-0.1266(4)$ & $0.0426(8)$ \\
\hline N6 & $0.3556(3)$ & $0.4933(3)$ & $-0.2144(5)$ & 0.0677 (13) \\
\hline $\mathrm{C} 1$ & $0.1363(2)$ & $0.60913(18)$ & $-0.0521(5)$ & $0.0328(8)$ \\
\hline $\mathrm{C} 2$ & $0.2207(2)$ & $0.62761(18)$ & $-0.0143(5)$ & $0.0322(8)$ \\
\hline C3 & $0.2471(2)$ & 0.67741 (19) & $75(5)$ & 0.0349 (8) \\
\hline $\mathrm{C} 4$ & $2(2)$ & (19) & & $5(8)$ \\
\hline H4 & 0.216392 & 0.737577 & -0.319905 & $0.044^{\star}$ \\
\hline $\mathrm{C} 5$ & $0.1146(3)$ & $0.68336(19)$ & $-0.2876(5)$ & $0.0361(8)$ \\
\hline C6 & $0.0844(2)$ & 0.63690 (19) & $-0.1874(5)$ & $0.0350(8)$ \\
\hline H6 & 0.028116 & 0.623734 & -0.210636 & $0.042^{*}$ \\
\hline $\mathrm{C} 7$ & $0.0994(2)$ & $0.56606(18)$ & $0.0611(5)$ & $0.0325(8)$ \\
\hline $\mathrm{H} 7$ & 0.040537 & 0.564193 & 0.051346 & $0.039^{\star}$ \\
\hline $\mathrm{C} 8$ & $0.0943(2)$ & $0.49533(19)$ & $0.2959(5)$ & $0.0355(8)$ \\
\hline $48 \mathrm{~A}$ & 0.096260 & 0.448933 & 0.273255 & $0.043^{*}$ \\
\hline H8B & 0.035481 & 0.508991 & 0.279128 & $0.043^{*}$ \\
\hline $\mathrm{C} 9$ & $0.1325(3)$ & $0.5092(2)$ & $0.4826(6)$ & $0.0488(11)$ \\
\hline H9A & 0.086820 & 0.518868 & & 0.059 * \\
\hline H9B & & & & $.059^{*}$ \\
\hline C10 & $0.1937(3)$ & $0.5628(2)$ & $0.5046(6)$ & 487 (11) \\
\hline $\mathrm{H} 10 \mathrm{~A}$ & 0.167669 & 0.601304 & 0.447785 & $0.058^{*}$ \\
\hline $10 B$ & 0.206682 & 366 & 3934 & $0.058^{*}$ \\
\hline C11 & $0.3343(3)$ & $0.6009(3)$ & $0.4743(6)$ & $0.0525(11)$ \\
\hline $\mathrm{H} 11 \mathrm{~A}$ & 0.335212 & 0.613296 & 0.596492 & $0.063^{*}$ \\
\hline $\mathrm{H} 11 \mathrm{~B}$ & 0.317119 & 0.638722 & 0.402972 & $0.063^{*}$ \\
\hline C12 & $0.4200(3)$ & $0.5826(3)$ & $0.4442(6)$ & $0.0541(12)$ \\
\hline $\mathrm{H} 12 \mathrm{~A}$ & 0.438148 & 0.546369 & 0.520901 & $0.065^{*}$ \\
\hline $\mathrm{H} 12 \mathrm{~B}$ & 0.457501 & 0.618895 & 0.478634 & $0.065^{\star}$ \\
\hline C13 & 0.4309 (3) & $0.5640(2)$ & $0.2608(5)$ & $0.0416(9)$ \\
\hline $\mathrm{H} 13 \mathrm{~A}$ & 0.414041 & 0.599564 & 180935 & $0.050^{*}$ \\
\hline H13B & & & & $0.050^{*}$ \\
\hline C14 & $0.4129(2)$ & $0.4536(2)$ & $0.2032(5)$ & 0378 (9) \\
\hline H14 & 0.471080 & 0.453426 & 0.197924 & $0.045^{*}$ \\
\hline C15 & $0.3711(2)$ & $0.3925(2)$ & $0.1959(5)$ & $0.0382(9)$ \\
\hline C16 & $0.4164(3)$ & $0.3376(2)$ & $0.1652(5)$ & 0.0426 (9) \\
\hline $\mathrm{H} 16$ & 0.470606 & 0.341411 & 0.132326 & $0.051^{*}$ \\
\hline $\mathrm{C} 17$ & $0.3825(3)$ & $0.2787(2)$ & $0.1826(6)$ & $0.0442(10)$ \\
\hline C18 & $0.3039(3)$ & $0.2717(2)$ & $0.2326(6)$ & $0.0440(10)$ \\
\hline H18 & 0.281083 & 0.230470 & 0.244834 & $0.053^{*}$ \\
\hline C19 & $0.2600(2)$ & & $0.2639(5)$ & $0.0384(9)$ \\
\hline $\mathrm{C} 20$ & $0.2900(2)$ & 0.38790 (19) & $0.2461(5)$ & 0.0344 (8) \\
\hline
\end{tabular}

Table 2: (continued)

\begin{tabular}{lrrrr}
\hline Atom & $\boldsymbol{x}$ & $\boldsymbol{y}$ & $\boldsymbol{z}$ & $\boldsymbol{U}_{\text {iso }} \boldsymbol{U}_{\text {eq }}$ \\
\hline 03 & $0.09136(19)$ & $0.42434(16)$ & $-0.1247(5)$ & $0.0555(8)$ \\
H3 & 0.135219 & 0.445995 & -0.110078 & $0.083^{*}$ \\
C21 & $0.1106(3)$ & $0.3611(2)$ & $-0.1612(7)$ & $0.0587(13)$ \\
H21A & 0.132326 & 0.359242 & -0.272361 & $0.088^{*}$ \\
H21B & 0.060295 & 0.334921 & -0.166228 & $0.088^{*}$ \\
H21C & 0.152491 & 0.344889 & -0.070615 & $0.088^{*}$ \\
\hline
\end{tabular}

\section{Comment}

Since the $\mathrm{N}$ atoms in $-\mathrm{C}=\mathrm{N}-$ in Schiff bases have the advantages of a high affinity and coordination ability for different transition metal ions, Schiff base ligands can construct versatile functional complexes, and the design and synthesis of Schiff base complexes arouse great interest $[5,6]$. Structures with corresponding halogenated ligands are still rare [7-9]. As a part of our current research the title complex was studied.

The Co(III) ion adopts a distorted octahedral geometry with a $\mathrm{CoN}_{4} \mathrm{O}_{2}$ core in the title compound. The environment around $\mathrm{Co}(\mathrm{III})$ comprises an azide ion and a nitrogen atom at the apical position and two nitrogen atoms and two oxygen atoms in the basal plane of which all the nitrogen and oxygen atoms (N1, N3, 01, O2) are from the pentadentate Schiff base ligand. Not surprisingly, the $\mathrm{Co}-\mathrm{N}$ bond distances are longer than the $\mathrm{Co}-\mathrm{O}$ bonds in the basal plane. The bond angles range from $87.34(13)^{\circ}$ to $179.43(14)^{\circ}$ in the title compound and are all in the expected ranges $[10,11]$.

Author contributions: All the authors have accepted responsibility for the entire content of this submitted manuscript and approved submission.

Research funding: This work was supported by Fund for Less Developed National Nature Science Foundation of China (Nos. 31760257, 21761017) as well as the Joint Basic Research Program (partial) of Yunnan Provincial Undergraduate Universities (2017FH001-002), the Program for Innovative Research Team (in Science and Technology) in Universities of Yunnan Province (IRTSTYN), the Recruitment Program of Yunnan Province Experts Provincial Young Talents (2019HB098) and the Ten-Thousand Talents Program of Yunnan Province (YNWR-QNBJ-2018-273).

Conflict of interest statement: The authors declare no conflicts of interest regarding this article. 


\section{References}

1. Agilent Technologies. CrysAlis ${ }^{\mathrm{PRO}}$ Software system; Agilent Technologies UK Ltd: Oxford, UK, 2015.

2. Dolomanov O. V., Bourhis L. J., Gildea R. J., Howard J. A. K., Puschmann H. OLEX2: a complete structure solution, refinement and analysis program. J. Appl. Crystallogr. 2009, 42, 339-341.

3. Sheldrick G. M. A short history of SHELX. Acta Crystallogr. 2008, A64, 112-122.

4. Sheldrick G. M. Crystal structure refinement with SHELXL. Acta Crystallogr. 2015, C71, 3-8.

5. Miyasaka H., Saitoh A., Abe S. Magnetic assemblies based on Mn(III) salen analogues. Coord. Chem. Rev. 2007, 251, 2622-2664.

6. Kachi-Terajima C., Ishii R., Tojo Y., Fukuda M., Kitagawa Y., Asaoka M., Miyasaka H. Ferromagnetic exchange coupling in a family of MnIII salen-type Schiff-base out-of-plane dimers. J. Phys. Chem. C 2017, 121, 12454-12468.
7. Ghosh M., Mandal S., Fleck M., Saha R., Rizzoli C., Bandyopadhyay D. Synthesis, crystal structure, and antimicrobial activity of a series of cobalt(III) Schiff base complexes. J. Coord. Chem. 2018, 71, 4180-4193.

8. Wu Q., Tang Y., Zi Q. Synthesis, crystallographic structure, Hirshfeld surface analysis and DFT calculations of two salen-type halogenated Schiff- base Ni(II) complexes. Polyhedron 2019, 166, 123-129.

9. Gomes L., Pinho D., Freire C., de Castro B. (1,4-Dioxane-O) $\left\{3,3^{\prime}, 5,5^{\prime}\right.$-tetrachloro-2, $2^{\prime}$-[4-methyl-4-azaheptane1,7-diylbis(nitrilomethyl- idyne- $N$ )]diphenolato- $O, O^{\prime}$ \}nickel(II),

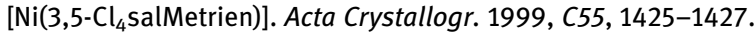

10. Mandal S., Layek M., Saha R., Rizzoli C., Bandyopadhyay D. Synthesis, crystal structure and antibacterial activity of four mononuclear Schiff base complexes of copper(II) and nickel(II). Transition Met. Chem. 2021, 46, 9-16.

11. Li T., Xu X., Huang M., Wang M., Wu Q. Crystal structure of tris(azido- $\left.{ }^{1} N\right)$-( $N$-(2-aminoethyl)- $N$-methyl-1,3-propanediamine$\left.\mathrm{K}^{3} N, N^{\prime}, N^{\prime \prime}\right)$ cobalt(III), $\mathrm{C}_{7} \mathrm{H}_{19} \mathrm{CoN}_{12}$. Z. Kristallogr. N. Cryst. Struct. 2021, 236, 357-358. 